\title{
CLICK REACTION ASSISTED SYNTHESIS OF HETEROCYCLIC LIQUID CRYSTALS AND ITS CHARACTERIZATION
}

\author{
T.S.Boopathi ${ }^{*}$ and S.Senthil ${ }^{2}$ \\ ${ }^{1}$ Dept of Sciences, Amrita School of Engineering, Coimbatore, Amrita Vishwa Vidyapeetham, \\ Amrita University, India \\ ${ }^{2}$ Department of chemistry, Government Arts College, Salem, India. \\ *E-mail: ts_boopathi@cb.amrita.edu
}

\begin{abstract}
The synthesis of 1,4-disubstituted-[1,2,3]-triazoles involving the addition of aryl azide to terminal acetylenes and ethyl acetoacetate are employed in the preparation of a series of non-linear mesogens having ester and imine as linking units. The structures of final compounds and their intermediates are characterized by FTIR, ${ }^{1} \mathrm{HNMR}$ and ${ }^{13}$ CNMR spectroscopy. Optical microscopy and differential scanning calorimetry confirm structural information of liquid crystal phases of mesogens. The final compounds reveal preferentially well-defined threaded nematic phases. In addition, they show weak fluorescence in solution with poor quantum yields and a Stokes shift of around $70 \mathrm{~nm}$. Keywords: Liquid crystal, heterocycle, triazole, click reaction, mesophase, nematic, kink effect.
\end{abstract}

(C) RASĀYAN. All rights reserved

\section{INTRODUCTION}

Liquid crystals are unique in their uses due to their excellent optical properties ${ }^{1}$. During the past decade liquid crystalline compounds having heterocyclic moieties plays a vital role in the design and preparation of highly developed functional materials ${ }^{2}$. In common, liquid crystals have initiated a tremendous change in technological applications, as of their more widespread use in flat-panel displays to their use as soft materials in the course of supramolecular assembly ${ }^{3}$. The center core units, linking moieties and terminal chains play a crucial role in designing a novel calamitic liquid crystals as superior functional material ${ }^{4,5,6}$. Owing to the non-linearity and less mesogenic stability of the six-membered rings ${ }^{7,8,9}$, researchers focused on the synthesis of liquid crystalline compounds possessing five-membered heterocyclic rings ${ }^{10}$.

In particular, [1,2,3]-triazoles like many other five-membered heterocyclic compounds have gained tremendous impetus in the field of materials chemistry ${ }^{11,12}$ organic chemistry ${ }^{13}$, supramolecular chemistry $^{14}$ and drug discovery ${ }^{15-17}$. One of the reason is most of the usual hetero atoms introduced are more polarizable than carbon $^{18,19}$. In addition, the hetero atoms have a strong upshot on the angle of rotation flanked by its fragments, the planarity and hence on the stability of mesophases and the temperatures of phase transitions ${ }^{20,21}$.

The [1,2,3]-triazole prepared by 'click chemistry' reaction as coined by Sharpless et al. ${ }^{22}$, has received explosive attention because of the inertness towards oxidizing, reducing and hydrolytic conditions at high temperatures. In addition, it has a good hydrogen bond accepting ability and enhances molecular anisotropy which is a prerequisite for achieving liquid crystalline materials. The well-accepted mechanism of "click chemistry" reaction is the Huisgen [2+3] dipolar cycloaddition reaction between an organic azide and terminal alkyne ${ }^{23-26}$. The regioselectivity is extensively controlled by employing the catalytic amount of $\mathrm{Cu}(\mathrm{I})$ salts $^{27}$. In this regard, this article deals with a series of mesogens possessing 1,4disubstituted-[1,2,3]-triazole having Schiff's base and ester units. 


\section{RASĀYAN J. Chem.}

Vol. 10 | No. 1 |136-148 | January - March | 2017

\section{Materials}

\section{EXPERIMENTAL}

$\mathrm{N}, \mathrm{N}$-dimethyl formamide (DMF), ethanol, chloroform, dichloro methane, acetonitrile and other solvents are used. 4-acetamido phenol, propargyl alcohol, potassium carbonate, tetra butyl ammonium bromide(TBAB), copper iodide(CuI), potassium iodide(KI), acetyl acetone, sodium metal, ethyl acetoacetate, naphthalene diol, quinol, p-toluene sulphonic acid, biphenyl diol, dicyclohexylcarbodiimide(DCC), 4-(dimethyl amino)pyridine(DMAP) and hydrated hydrazine are used as received. By the simple SN2 mechanism the 1-bromohexane is prepared from hydrobromic acid and 1hexanol using benzene as per reported procedure ${ }^{28}$.

\section{Techniques and Characterization}

The moisture sensitive reactions are carried in sealed rubber septa and reagents are injected through a syringe. The progress of the reaction is monitored by a Thin layer chromatography with Merck pre-coated silica gel $60 \mathrm{~F}_{254}$ Aluminium sheets as an adsorbent, chloroform, ethyl acetate, 1:1 hexane and ethyl acetate as the mobile phase and iodine, UV light as the visualizing agent. Infrared spectra are recorded by Nicolet iS10 FTIR Spectrometer using $1 \% \mathrm{KBr}$ disc and nuclear magnetic resonance spectra using Bruker Advance III 500MHZ (AV500) multi nuclei solution NMR in a $\mathrm{CDCl}_{3}$ solvent with TMS as an internal standard for both ${ }^{1} \mathrm{HNMR}$ and ${ }^{13} \mathrm{CNMR}$ spectra (chemical shift in $\delta \mathrm{ppm}$ ). With NETZSCH DSC 204, the thermal characterization involving energy effects like phase transitions, thermal stability, transition temperatures etc., are studied by heating and cooling rates of $10^{\circ} \mathrm{C}$ rise per minute under air atmosphere. Hot stage optical polarizing microscopy is performed on a Euromex polarizing microscope equipped with a Linkem HFS91 hot stage and a TP93 thermal controller. To obtain micrographs small quantity of the synthesized target compound is sandwiched between two thin glass cover slips and the anisotropic behavior is observed by heating and/or cooling at the rates of $5^{\circ} \mathrm{C} / \mathrm{min}$. Using Nikon FM10 camera, the photographs are taken and exposed on a Konica film.

\section{Synthesis of 4-hexyloxy phenyl acetamide (1)}

To a solution of 4-acetamidophenol $(0.099 \mathrm{~mol}, 15 \mathrm{~g})$ dissolved in DMF, 1-bromohexane (0.109mol, $18 \mathrm{ml})$, potassium carbonate $(0.16 \mathrm{~mol}, 22 \mathrm{~g})$, TBAB $(2$ weight $\%, 0.6 \mathrm{~g})$ and potassium iodide (10 weight $\%$, $5 \mathrm{~g}$ ) are added. After being refluxed in an oil bath at $90-95^{\circ} \mathrm{C}$ for 12 hours, the mixture is poured into crushed ice, filtered and dried. The yield of the crude white solid (1) is $67 \%$.

\section{$\operatorname{FTIR}\left(\mathrm{KBr}, \mathbf{c m}^{-1}\right)$}

3283(m), 3253(m) (d, amide $-\mathrm{NH}_{2}$, str), 3074(m) (Ar $-\mathrm{C}-\mathrm{H}$, str), 2930(s) (aliphatic $-\mathrm{C}-\mathrm{CH}_{3}$, str), 2866(s) (aliphatic $-\mathrm{CH}_{2}-$, str), 1659(s) (amide $-\mathrm{C}=\mathrm{O}$, str), 1603(m), 1543(m) (Ar C=C, str), 1473(s) (aliphatic $-\mathrm{C}-\mathrm{CH}_{2}-$, bend), 1385(s) (aliphatic $-\mathrm{C}-\mathrm{CH}_{3}$, str), 1371(s) (-C-N, str), 1240(s) ( $\mathrm{Ar}-\mathrm{O}-\mathrm{CH}_{2}-$, str), $1029(\mathrm{~m})\left(-\mathrm{O}-\mathrm{CH}_{2}-\right.$, bend), 830(s) (Ar para disubstitution, bend).

\section{${ }^{1} \mathbf{H}-\mathbf{N M R}\left[\mathrm{CDCl}_{3}\right] \boldsymbol{\delta}(\mathbf{p p m})$}

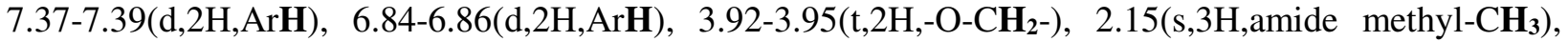
1.76-1.80(m,2H,-O- $\left.\mathrm{CH}_{2}-\mathrm{CH}_{2}-\right), 1.44-1.47\left(\mathrm{~m}, 2 \mathrm{H},-\mathrm{CH}_{2}-\mathrm{CH}_{2}-\mathrm{CH}_{2}-\mathrm{CH}_{3}\right), 1.33-1.36\left(\mathrm{~m}, 4 \mathrm{H},-\mathrm{CH}_{2}-\mathrm{CH}_{2}-\mathrm{CH}_{3}\right)$, $0.90-0.93\left(\mathrm{t}, 3 \mathrm{H},-\mathrm{CH}_{2}-\mathrm{CH}_{2}-\mathrm{CH}_{3}\right)$

\section{${ }^{13} \mathrm{C}-\mathrm{NMR}\left[500 \mathrm{MHz}, \mathrm{CDCl}_{3}\right] \boldsymbol{\delta}(\mathbf{p p m})$}

168.35(Quaternary carbon of carbonyl in amide), 156.05(aromatic -C-O-), 130.79(aromatic -C-N-), $121.95,114.77$ (aromatic $=\mathbf{C H}-), 68.31\left(-\mathrm{O}-\mathrm{CH}_{2}-\right), 31.58\left(-\mathrm{O}-\mathrm{CH}_{2}-\mathrm{CH}_{2}-\right), 29.24\left(-\mathrm{CH}_{2}-\mathrm{CH}_{2}-\mathrm{CH}_{2}-\mathrm{CH}_{3}\right)$, 25.70(- $\left.\mathrm{CH}_{2}-\mathrm{CH}_{2}-\mathrm{CH}_{3}\right), 22.59\left(-\mathrm{CH}_{2}-\mathrm{CH}_{3}\right), 14.02\left(-\mathrm{CH}_{2}-\mathrm{CH}_{3}\right)$.

\section{Synthesis of 4-hexyloxy phenyl amine (2)}

In a round-bottomed flask, charged (1) $(0.0426 \mathrm{~mol}, 10 \mathrm{~g})$ in ethanol $(50 \mathrm{ml})$, hydrochloric acid $(50 \mathrm{ml})$ and refluxed for 6 hours. The completion of the reaction is monitored by TLC. After completion the reaction mixture (2) is used as such for the next stage. The yield is $95 \%$. 


\section{Synthesis of 4-hexyloxy phenyl azide (3)}

The above-prepared amine $(0.052 \mathrm{~mol}, 10 \mathrm{~g})$ taken in a $500 \mathrm{ml}$ beaker equipped with a stirrer and a thermometer is cooled to $0-10^{\circ} \mathrm{C}$ under freezing mixture. Likewise, the sodium nitrite $(0.061 \mathrm{~mol}, 4.2 \mathrm{~g})$ and sodium azide $(0.078 \mathrm{~mol}, 5.05 \mathrm{~g})$ in water are also cooled separately. A solution of sodium nitrite in water is added drop wise to the $500 \mathrm{ml}$ beaker followed by sodium azide while maintaining the temperature $0-10^{\circ} \mathrm{C}$ strictly. After the reaction has completed, the brown oily layer is isolated using chloroform, filtered over calcium chloride and concentrated under high vacuum. The recovered product (3) is used as such for the next subsequent stages. The yield is $85 \%$.

\section{$\operatorname{FTIR}\left(\mathrm{KBr}, \mathbf{c m}^{-1}\right)$}

3074(m) (Ar -C-H, str), 2928(s) (aliphatic $-\mathrm{C}-\mathrm{CH}_{3}$, str), 2856(s) (aliphatic $-\mathrm{CH}_{2}-$, str), 2111(s) $\left(\mathrm{N}_{3}\right.$, str), 1584(m), 1503(m) (Ar C=C, str), 1469(s) (aliphatic $-\mathrm{C}-\mathrm{CH}_{2}-$, bend), 1387(s) (aliphatic $-\mathrm{C}-\mathrm{CH}_{3}$, str), 1371(s) (-C-N, str), 1244(s) ( $\mathrm{Ar}-\mathrm{O}-\mathrm{CH}_{2}-$, str), 1029(m) $\left(-\mathrm{O}-\mathrm{CH}_{2-}\right.$, bend), 825(s) (Ar para disubstitution, bend).

\section{Synthesis of 5-methyl-1-[4-hexyloxy phenyl]-1H-[1,2,3]-triazole-4-carboxylic acid (4)}

To the ethanol taken in a round bottomed flask kept under ice cold water added the sodium metal $(5 \mathrm{~g})$ slowly with constant stirring. After the complete addition of $\mathrm{Na}$ metal, ethyl acetoacetate $(0.053 \mathrm{~mol}$, $6.84 \mathrm{~g})$ is added followed by 4-hexyloxy azido phenol (3) $(0.044 \mathrm{~mol}, 9.6 \mathrm{~g})$ and the reaction mixture is refluxed for 12 hours at $90^{\circ} \mathrm{C}$. The solid obtained after subsequent concentration, filtration from ice cold water is dried and weighed. The yield of half white solid is $60 \%$.

\section{$\operatorname{FTIR}\left(\mathrm{KBr}, \mathbf{c m}^{-1}\right)$}

3448(b) (acid -OH, str), 3073(m) (Ar -C-H, str), 2940(s) (aliphatic $-\mathrm{C}-\mathrm{CH}_{3}$, str), 2869(s) (aliphatic $\mathrm{CH}_{2-}$, str), 1692(s) (acid $\left.-\mathrm{C}=\mathrm{O}, \mathrm{str}\right), 1608(\mathrm{~m}), 1566(\mathrm{~m})$ ( $\left.\mathrm{Ar} \mathrm{C}=\mathrm{C}, \mathrm{str}\right), 1515(\mathrm{~s})(-\mathrm{N}=\mathrm{N}-$ in triazole ring, str), 1446(s) (aliphatic $-\mathrm{C}-\mathrm{CH}_{2}-$, bend), 1398(s) (aliphatic $\left.-\mathrm{C}-\mathrm{CH}_{3}, \mathrm{str}\right), 1247$ (s) ( $\mathrm{Ar}-\mathrm{O}-\mathrm{CH}_{2}-$, str), $1116(\mathrm{~m})\left(\right.$ acid $-\mathrm{C}-\mathrm{O}-$, str), 1026(m) $\left(-\mathrm{O}-\mathrm{CH}_{2-}\right.$, bend), 836(s) (Ar para disubstitution, bend)

\section{${ }^{1} \mathbf{H}-\mathbf{N M R}\left[\mathrm{CDCl}_{3}\right] \boldsymbol{\delta}(\mathbf{p p m})$}

7.35-7.38(d,2H,ArH $), 7.05-7.08(\mathrm{~d}, 2 \mathrm{H}, \mathrm{ArH}), 4.03-4.06\left(\mathrm{t}, 2 \mathrm{H},-\mathrm{O}-\mathbf{C H}_{2}-\right), 2.60\left(\mathrm{~s}, 3 \mathrm{H},-\mathrm{CH}_{3}\right.$ attached to $\mathrm{C}=\mathrm{N}-$ in [1,2,3]-triazole ring), $1.81-1.87\left(\mathrm{~m}, 2 \mathrm{H},-\mathrm{O}-\mathrm{CH}_{2}-\mathrm{CH}_{2}-\right), 1.49-1.52\left(\mathrm{~m}, 2 \mathrm{H},-\mathrm{CH}_{2}-\mathrm{CH}_{2}-\mathrm{CH}_{2}-\mathrm{CH}_{3}\right)$, $1.35-1.39\left(\mathrm{~m}, 4 \mathrm{H},-\mathrm{CH}_{2}-\mathrm{CH}_{2}-\mathrm{CH}_{3}\right), 0.92-0.95\left(\mathrm{t}, 3 \mathrm{H},-\mathrm{CH}_{2}-\mathrm{CH}_{2}-\mathrm{CH}_{3}\right)$

${ }^{13} \mathrm{C}-\mathrm{NMR}\left[\mathbf{5 0 0 M H z}, \mathrm{CDCl}_{3}\right] \boldsymbol{\delta}$ (ppm)

164.74(Quaternary carbon of carbonyl in acid), 160.44(aromatic -C-O-), 139.91 (aromatic -C-N-), 135.76, 127.79(-C=N- in [1,2,3]-triazole ring), 126.70, 115.31(aromatic = $\mathbf{C H}-)$, 68.56(-O- $\left.\mathbf{C H}_{2}-\right), 31.53(-$ $\left.\mathrm{O}-\mathrm{CH}_{2}-\mathrm{CH}_{2}-\right), 29.06\left(-\mathrm{CH}_{2}-\mathrm{CH}_{2}-\mathrm{CH}_{2}-\mathrm{CH}_{3}\right), 25.66\left(-\mathrm{CH}_{2}-\mathrm{CH}_{2}-\mathrm{CH}_{3}\right), 22.58\left(-\mathrm{CH}_{2}-\mathrm{CH}_{3}\right), 14.01\left(-\mathrm{CH}_{2}-\mathrm{CH}_{3}\right)$, 9.99(- $-\mathrm{CH}_{3}$ attached to triazole ring)

\section{Synthesis of 1-[4-hexyloxy phenyl]-1H-[1,2,3]-triazole-4-carbinol (5)}

The 4-hexyloxy azido phenol (3) $(0.039 \mathrm{~mol}, 8.47 \mathrm{~g})$ taken in a $250 \mathrm{ml}$ round-bottomed flask containing magnetic stirrer is charged with propargyl alcohol $(0.058 \mathrm{~mol}, 3.25 \mathrm{ml}), \mathrm{CuI}(10$ weight $\%, 1.17 \mathrm{~g}), \mathrm{Et}_{3} \mathrm{~N}$ $(0.039 \mathrm{~mol}, 3.91 \mathrm{ml})$ and acetonitrile. The reaction mass is continuously stirred at room temperature for 8hours. The reaction mixture after completion is filtered and concentrated. The yield of the crude solid is $60 \%$.

\section{FTIR (KBr, cm $\left.{ }^{-1}\right)$}

3221(s) (alcohol -OH, str), 3078(m) (Ar-C-H, str), 2934(s) (aliphatic -C- $\mathrm{CH}_{3}$, str), 2867(s) (aliphatic $\mathrm{CH}_{2}-$, str), 1610(m) (Ar C=C, str), 1517(s) $\left(-\mathrm{N}=\mathrm{N}-\right.$ in triazole ring, str), 1469 (s) (aliphatic $-\mathrm{C}-\mathrm{CH}_{2}-$, bend), 1394(s) (aliphatic $-\mathrm{C}-\mathrm{CH}_{3}$, str), 1252(s) ( $\mathrm{Ar}-\mathrm{O}-\mathrm{CH}_{2-}$, str), 1050(b) (1 ${ }^{\circ}$ alcohol), 1017(m) (-O$\mathrm{CH}_{2-}$, bend), 834(s) (Ar para disubstitution, bend). 
RASĀYAN $J$. Chem.

Vol. 10 | No. 1 |136-148 | January - March | 2017

${ }^{1} \mathrm{H}-\mathrm{NMR}\left[\mathrm{CDCl}_{3}\right] \boldsymbol{\delta}(\mathbf{p p m})$

8.46(s,1H,-CH of triazole ring), 7.57-7.60(d,2H, $\mathrm{ArH}), 6.98-7.01(\mathrm{~d}, 2 \mathrm{H}, \mathrm{ArH}), 4.88\left(\mathrm{~s}, 2 \mathrm{H},-\mathrm{CH} \mathbf{H}_{2}-\mathrm{OH}\right), 3.98-$

$4.01\left(\mathrm{t}, 2 \mathrm{H},-\mathrm{O}-\mathrm{CH}_{2}-\right), 2.99\left(\mathrm{~s}, 1 \mathrm{H},-\mathrm{CH}_{2}-\mathrm{OH}\right), \quad 1.78-1.83\left(\mathrm{~m}, 2 \mathrm{H},-\mathrm{O}-\mathrm{CH}_{2}-\mathrm{CH}_{2}-\right), 1.44-1.49\left(\mathrm{~m}, 2 \mathrm{H},-\mathrm{CH}_{2}-\mathrm{CH}_{2}-\right.$

$\left.\mathrm{CH}_{2}-\mathrm{CH}_{3}\right), 1.33-1.37\left(\mathrm{~m}, 4 \mathrm{H},-\mathrm{CH}_{2}-\mathrm{CH}_{2}-\mathrm{CH}_{3}\right), 0.90-0.93\left(\mathrm{t}, 3 \mathrm{H},-\mathrm{CH}_{2}-\mathrm{CH}_{2}-\mathrm{CH}_{3}\right)$

${ }^{13} \mathrm{C}-\mathrm{NMR}\left[500 \mathrm{MHz}, \mathrm{CDCl}_{3}\right] \boldsymbol{\delta}(\mathbf{p p m})$

159.50(aromatic $-\mathbf{C}-\mathrm{O}-$ ), 130.25 (aromatic $-\mathbf{C}-\mathrm{N}-$ ), 122.22, 115.31(aromatic $=\mathbf{C H}-)$, 68.49(-O- $\mathbf{C H}_{2}-$ ), $31.55\left(-\mathrm{O}-\mathrm{CH}_{2}-\mathrm{CH}_{2}-\right)$, 29.12(- $\left.\mathrm{CH}_{2}-\mathrm{CH}_{2}-\mathrm{CH}_{2}-\mathrm{CH}_{3}\right), \quad 25.67\left(-\mathrm{CH}_{2}-\mathrm{CH}_{2}-\mathrm{CH}_{3}\right), \quad 22.59\left(-\mathrm{CH}_{2}-\mathrm{CH}_{3}\right), \quad 14.02(-$ $\left.\mathrm{CH}_{2}-\mathrm{CH}_{3}\right)$.

\section{Synthesis of 1-[4-hexyloxy phenyl]-1H-[1,2,3]-triazole-4-carbaldehyde (6)}

The above-synthesized carbinol (5) is converted into aldehyde (6) as per reported procedure ${ }^{29}$. According to the procedure, the jones reagent ${ }^{30}$ is added to the kieselguhr $(26 \mathrm{~g})$ under vigorously stirring condition until the orange powders are produced. To this a solution of alcohol $(5)(0.011 \mathrm{~mol}, 3 \mathrm{~g})$ in dichloro methane $(10 \mathrm{ml})$ is added slowly under a stirring condition at room temperature for 10 minutes. The reaction progress is monitored by TLC using hexane-ethyl acetate (4:2) as eluent. The solid is filtered and washed with dichloro methane. The filtrates are concentrated and the pure crystals are obtained using column chromatography with chloroform as developing solvent. The combined extracts are concentrated to get the crude white crystalline solid (6). The yield is $95 \%$.

(The jones reagent is prepared by dissolving $\mathrm{CrO}_{3}(2 \mathrm{~g})$ in $\mathrm{H}_{2} \mathrm{SO}_{4}(2 \mathrm{ml})$ and then water $(6 \mathrm{ml})$ added slowly).

\section{$\operatorname{FTIR}\left(\mathrm{KBr}, \mathrm{cm}^{-1}\right)$}

3041(m) (Ar $-\mathrm{C}-\mathrm{H}, \mathrm{str}), 2935$ (s) (aliphatic $-\mathrm{C}-\mathrm{CH}_{3}$, str), 2872(s) (aliphatic- $\mathrm{CH}_{2-}$, str), 2852(w), 2772(w) (aliphatic $-\mathrm{C}-\mathrm{H}$ in aldehyde, str), 1690(s) (aldehyde $\mathrm{C}=\mathrm{O}$, str), 1609(m) (Ar $-\mathrm{C}=\mathrm{C}-$, str), 1525(s) ( $-\mathrm{N}=\mathrm{N}-$ in triazole ring, str), 1474(s) (aliphatic $-\mathrm{C}-\mathrm{CH}_{2}-$, bend), 1399(s) (aliphatic $-\mathrm{C}-\mathrm{CH}_{3}$, str), 1259(s) ( $\left.\mathrm{Ar}-\mathrm{O}-\mathrm{CH}_{2-}, \mathrm{str}\right), 1026(\mathrm{~m})\left(-\mathrm{O}-\mathrm{CH}_{2-}\right.$, bend), 827(s) (Ar para disubstitution, bend).

\section{${ }^{1} \mathrm{H}-\mathrm{NMR}\left[\mathrm{CDCl}_{3}\right] \boldsymbol{\delta}(\mathbf{p p m})$}

10.22(s,1H,-CHO), 8.46(s,1H,-CH of [1,2,3]-triazole ring), 7.65-7.66(d,2H,ArH), 7.04-7.06(d,2H,ArH), 4.02-4.04(t, $\left.2 \mathrm{H},-\mathrm{O}-\mathrm{CH}_{2}-\right), 1.80-1.86\left(\mathrm{~m}, 2 \mathrm{H},-\mathrm{O}-\mathrm{CH}_{2}-\mathrm{CH}_{2}-\right), 1.48-1.51\left(\mathrm{~m}, 2 \mathrm{H},-\mathrm{CH}_{2}-\mathrm{CH}_{2}-\mathrm{CH}_{2}-\mathrm{CH}_{3}\right), \quad 1.36-$ $1.37\left(\mathrm{~m}, 4 \mathrm{H},-\mathrm{CH}_{2}-\mathrm{CH}_{2}-\mathrm{CH}_{3}\right), 0.93-0.94\left(\mathrm{t}, 3 \mathrm{H},-\mathrm{CH}_{2}-\mathrm{CH}_{3}\right)$

${ }^{13} \mathrm{C}-\mathrm{NMR}\left[500 \mathrm{MHz}, \mathrm{CDCl}_{3}\right] \delta(\mathrm{ppm})$

185.12(Quaternary carbon of carbonyl in aldehyde), 160.20(aromatic -C-O-), 148.03(aromatic -C-N-), $129.23,123.07$ (=C- in triazole ring), $122.45,115.53$ (aromatic $=\mathbf{C H}-)$, 68.57(-O- $\left.\mathbf{C H}_{2-}\right), 31.53\left(-\mathrm{O}-\mathrm{CH}_{2}-\right.$ $\left.\mathrm{CH}_{2}-\right), 29.07\left(-\mathrm{CH}_{2}-\mathrm{CH}_{2}-\mathrm{CH}_{2}-\mathrm{CH}_{3}\right), 25.65\left(-\mathrm{CH}_{2}-\mathrm{CH}_{2}-\mathrm{CH}_{3}\right), 22.57\left(-\mathrm{CH}_{2}-\mathrm{CH}_{3}\right), 14.01\left(-\mathrm{CH}_{2}-\mathrm{CH}_{3}\right)$.

Synthesis of 1,4-disubstituted-[1,2,3]-triazole containing Schiff's base as center core (IV)

A mixture of aldehyde (6) (0.00219 mol, 0.6g), hydrated hydrazine (0.0012 mol, 0.06g), p-toluene sulphonic acid and ethanol (for free stirring) are taken in a $100 \mathrm{ml} \mathrm{RB}$ equipped with a magnetic stirrer and stirred for 24 hours. The solid obtained after subsequent filtration, concentration is recrystallised from ethanol. The yield of a white solid is $80 \%$.

$\operatorname{FTIR}\left(\mathrm{KBr}, \mathbf{c m}^{-1}\right)$

3071(m) (Ar -C-H, str), 2931(s) (aliphatic $-\mathrm{C}-\mathrm{CH}_{3}, \mathrm{str}$ ), 2865(s) (aliphatic- $\mathrm{CH}_{2}-$, str), $1644(-\mathrm{C}=\mathrm{N}-$, str), 1594(m) (Ar C=C, str), 1522(s) (-N=N- in triazole ring, str), 1469(s) (aliphatic $-\mathrm{C}-\mathrm{CH}_{2}-$, bend), 1393(s) (aliphatic $-\mathrm{C}-\mathrm{CH}_{3}$, str), 1359(s) (-C-N, str), 1248(s) ( $\mathrm{Ar}-\mathrm{O}-\mathrm{CH}_{2}-$, str), 1041(m) (-O- $\mathrm{CH}_{2}-$, bend), 831(s) (Ar para disubstitution, bend).

${ }^{1} \mathrm{H}-\mathrm{NMR}\left[\mathrm{CDCl}_{3}\right] \boldsymbol{\delta}(\mathbf{p p m})$

8.85(s,2H,-CH=N- of imine unit), 8.46(s,2H,-CH=N- of [1,2,3]-triazole), 7.63-7.71(d,4H,ArH), 7.017.05(d,4H,ArH), 4.01-4.04(d,4H,-O- $\left.\mathbf{C H}_{2}-\right), 1.82-1.86\left(\mathrm{~m}, 4 \mathrm{H},-\mathrm{O}-\mathrm{CH}_{2}-\mathrm{CH}_{2}-\right), 1.48-1.50\left(\mathrm{~m}, 4 \mathrm{H},-\mathrm{CH}_{2}-\mathrm{CH}_{2-}\right.$ $\left.\mathrm{CH}_{2}-\mathrm{CH}_{3}\right), 1.37-1.38\left(\mathrm{~m}, 8 \mathrm{H},-\mathrm{CH}_{2}-\mathrm{CH}_{2}-\mathrm{CH}_{3}\right), 0.93-0.94\left(\mathrm{t}, 6 \mathrm{H},-\mathrm{CH}_{2}-\mathrm{CH}_{2}-\mathrm{CH}_{3}\right)$ 


\section{RASĀYAN J. Chem.}

Vol. 10 | No. 1 |136-148 | January - March | 2017

\section{${ }^{13} \mathrm{C}-\mathrm{NMR}\left[500 \mathrm{MHz}, \mathrm{CDCl}_{3}\right] \boldsymbol{\delta}(\mathbf{p p m})$}

159.83(aromatic -C-O-), 143.51, 134.55(aromatic $-\mathbf{C}-\mathrm{N}-$ ), 129.71, 124.25, 122.38(=C- in triazole ring) $122.28,122.11,115.34,115.43$ (aromatic $=\mathbf{C H}-), 120.33,118.36(=\mathbf{C H}-\mathrm{N}-$ in azo methine), 68.54, 68.49($\left.\mathrm{O}-\mathrm{CH}_{2}-\right), 31.55\left(-\mathrm{O}-\mathrm{CH}_{2}-\mathrm{CH}_{2}-\right), 29.10\left(-\mathrm{CH}_{2}-\mathrm{CH}_{2}-\mathrm{CH}_{2}-\mathrm{CH}_{3}\right), 25.66\left(-\mathrm{CH}_{2}-\mathrm{CH}_{2}-\mathrm{CH}_{3}\right), 22.59\left(-\mathrm{CH}_{2}-\mathrm{CH}_{3}\right)$, 14.02(- $\left.-\mathrm{CH}_{2}-\mathrm{CH}_{3}\right)$.

\section{Synthesis of 1, 4-disubstituted-[1,2,3]-triazole esters (I,II,III)}

To a solution containing acid $(0.00075 \mathrm{~mol}, 0.5 \mathrm{~g})$ and DMAP $(5-10$ weight $\%)$ in $\mathrm{CH}_{2} \mathrm{Cl}_{2}$, a solution of DCC (3equivalent) in dried $\mathrm{CH}_{2} \mathrm{Cl}_{2}$ is added. This solution is kept under stirring at room temperature. To this mixture, the 1,5-napthalene diol is added. The dicyclohexylurea formed during the reaction is filtered after 12 hours of stirring and the filtrate is concentrated. The 1,4-disubstituted-[1,2,3]-triazole ester containing naphthalene as the center core as pale brown solid (II) is collected after subsequent recrystallisation from ethanol. By employing the similar procedure, other 1,4-disubstituted-[1,2,3]-triazole esters containing quinol as white solid (I) and biphenyl as white solid (III) are prepared using quinol$0.00075 \mathrm{~mol}, 0.08258 \mathrm{~g}$ and biphenyl diol $-0.0009 \mathrm{~mol}, 0.168 \mathrm{~g}$. The yield of I, II and III are $80 \%$, 75\%, and $78 \%$ respectively.

\section{(I) $\quad \operatorname{FTIR}\left(\mathrm{KBr}, \mathrm{cm}^{-1}\right)$}

3071(m) (Ar -C-H, str), 2924(s) (aliphatic $-\mathrm{C}-\mathrm{CH}_{3}$, str), 2853(s) (aliphatic $-\mathrm{CH}_{2-}$, str), 1739(s) (ester $\mathrm{C}=\mathrm{O}, \mathrm{str}), 1606(\mathrm{~m})\left(\mathrm{Ar}-\mathrm{C}=\mathrm{C}-\right.$, str), 1513(s) $\left(-\mathrm{N}=\mathrm{N}-\right.$ in triazole ring, str), $1466(\mathrm{~s})$ (aliphatic $-\mathrm{C}-\mathrm{CH}_{2}-$, bend), 1385(s) (aliphatic $-\mathrm{C}-\mathrm{CH}_{3}$, str), 1247(s) (Ar-O- $\mathrm{CH}_{2}$, str), 1175(s) (ester -C-O-, str), 1095(m) ($\mathrm{O}-\mathrm{CH}_{2}-$, bend), 842(s) (Ar para disubstitution, bend).

\section{${ }^{\mathbf{1}} \mathrm{H}-\mathrm{NMR}\left[\mathrm{CDCl}_{3}\right] \boldsymbol{\delta} \mathbf{p p m}$}

7.37-7.40(m,8H,ArH and QuinolH), 7.07-7.09(d,4H,ArH), 4.04-4.07(d,4H-O-CH $\left.\mathbf{2}^{-}\right), 2.63\left(\mathrm{~s}, 6 \mathrm{H},-\mathbf{C H}_{3}\right.$ attached to $-\mathrm{C}=\mathrm{N}-$ in $[1,2,3]$-triazole ring $), 1.82-1.88\left(\mathrm{~m}, 4 \mathrm{H},-\mathrm{O}-\mathrm{CH}_{2}-\mathrm{CH}_{2}-\right), 1.50-1.51\left(\mathrm{~m}, 4 \mathrm{H},-\mathrm{CH}_{2}-\mathrm{CH}_{2}-\right.$ $\left.\mathrm{CH}_{2}-\mathrm{CH}_{3}\right), 1.38-1.39\left(\mathrm{~m}, 8 \mathrm{H},-\mathrm{CH}_{2}-\mathrm{CH}_{2}-\mathrm{CH}_{3}\right), 0.94-0.95\left(\mathrm{t}, 6 \mathrm{H},-\mathrm{CH}_{2}-\mathrm{CH}_{2}-\mathrm{CH}_{3}\right)$.

${ }^{13} \mathrm{C}-\mathrm{NMR}\left[500 \mathrm{MHz}, \mathrm{CDCl}_{3}\right] \mathbf{\delta p p m}$

160.45(carbonyl carbon in ester), 160.11(aromatic -C-O-), 147.95(aromatic -C-O-), 140.25, 135.66(aromatic $-\mathrm{C}-\mathrm{N}-)$, 127.86, 122.76( $=\mathbf{C}$ - in triazole ring) 126.74, 115.33(aromatic $=\mathbf{C H}-)$, 68.56(-O$\left.\mathrm{CH}_{2}-\right)$, 31.54(-O-CH$\left.{ }_{2}-\mathrm{CH}_{2}-\right)$, 29.69, 29.07(- $\left.\mathrm{CH}_{2}-\mathrm{CH}_{2}-\mathrm{CH}_{2}-\mathrm{CH}_{3}\right), 25.67\left(-\mathrm{CH}_{2}-\mathrm{CH}_{2}-\mathrm{CH}_{3}\right), 22.59\left(-\mathrm{CH}_{2}-\right.$ $\left.\mathrm{CH}_{3}\right), 14.02\left(-\mathrm{CH}_{2}-\mathrm{CH}_{3}\right), 10.07\left(-\mathrm{CH}_{3}\right.$ attached to triazole ring $)$.

\section{(II) FTIR $\left(\mathrm{KBr}, \mathrm{cm}^{-1}\right)$}

3073(m) (Ar $-\mathrm{C}-\mathrm{H}, \mathrm{str}), 2926$ (s) (aliphatic $-\mathrm{C}-\mathrm{CH}_{3}$, str), 2853(s) (aliphatic $-\mathrm{CH}_{2}-$, str), 1739(s) (ester $\mathrm{C}=\mathrm{O}$, str), 1603(m) (Ar C=C, str), 1513(s) $(-\mathrm{N}=\mathrm{N}-$ in [1,2,3]-triazole ring, str), 1466(s) (aliphatic $-\mathrm{C}-$ $\mathrm{CH}_{2-}$, bend), 1247(s) (Ar-O- $\mathrm{CH}_{2-}$, str), 1166(s) (ester -C-O-, str), 1095(m) (-O- $\mathrm{CH}_{2-}$, bend), 845(s) (Ar para disubstitution, bend).

\section{${ }^{1} \mathbf{H}-\mathrm{NMR}\left[\mathrm{CDCl}_{3}\right] \mathbf{\delta p p m}$}

7.93-7.95(d,2H,naphthaleneH), 7.81-7.82(d,2H,naphthaleneH), 7.47-7.49(t,2H,naphthaleneH), 7.407.42(m,4H,ArH), 7.08-7.10(d,4H,ArH), 4.05-4.07(d,4H,-O- $\left.\mathbf{C H}_{2}-\right), 2.66\left(\mathrm{~s}, 6 \mathrm{H},-\mathrm{CH}_{3}\right.$ attached to $-\mathrm{C}=\mathrm{N}-$ in [1,2,3]-triazole ring), $1.84-1.87\left(\mathrm{~m}, 4 \mathrm{H},-\mathrm{O}-\mathrm{CH}_{2}-\mathrm{CH}_{2}-\right), \quad 1.50-1.53\left(\mathrm{~m}, 4 \mathrm{H},-\mathrm{CH}_{2}-\mathrm{CH}_{2}-\mathrm{CH}_{2}-\mathrm{CH}_{3}\right), \quad 1.38-$ $1.39\left(\mathrm{~m}, 8 \mathrm{H},-\mathrm{CH}_{2}-\mathrm{CH}_{2}-\mathrm{CH}_{3}\right), 0.88-0.96\left(\mathrm{t}, 6 \mathrm{H},-\mathrm{CH}_{2}-\mathrm{CH}_{2}-\mathrm{CH}_{3}\right)$.

\section{${ }^{13} \mathrm{C}-\mathrm{NMR}\left[500 \mathrm{MHz}, \mathrm{CDCl}_{3}\right] \delta \mathrm{ppm}$}

160.46(carbonyl carbon in ester), 160.43(aromatic -C-O-), 148.10(aromatic -C-O-), 140.30, 135.74(aromatic -C-N-), 131.94, 129.28, 118.98(naphthalene carbon), 127.88, 122.13(=C- in triazole ring), 126.75, 115.34(aromatic =CH-), 68.57(-O- $\left.\mathrm{CH}_{2}-\right)$, 31.92, 31.55(-O- $\left.\mathrm{CH}_{2}-\mathbf{C H}_{2}-\right), 29.69,29.08\left(-\mathbf{C H}_{2}-\right.$ $\left.\mathrm{CH}_{2}-\mathrm{CH}_{2}-\mathrm{CH}_{3}\right), 25.67\left(-\mathrm{CH}_{2}-\mathrm{CH}_{2}-\mathrm{CH}_{3}\right), 22.59,22.69\left(-\mathrm{CH}_{2}-\mathrm{CH}_{3}\right), 14.02,14.11\left(-\mathrm{CH}_{2}-\mathrm{CH}_{3}\right), 10.08\left(-\mathrm{CH}_{3}\right.$ attached to triazole ring). 
RASĀYAN J. Chem.

Vol. 10 | No. 1 |136-148 | January - March | 2017

(III) $\operatorname{FTIR}\left(\mathrm{KBr}, \mathrm{cm}^{-1}\right)$

3072(m) (Ar -C-H, str), 2926(s) (aliphatic $-\mathrm{C}-\mathrm{CH}_{3}$, str), 2854(s) (aliphatic $-\mathrm{CH}_{2}-$, str), 1744(s) (ester $\mathrm{C}=\mathrm{O}$, str), 1606(m) (Ar C=C, str), 1515(s) $(-\mathrm{N}=\mathrm{N}-$ in [1,2,3]-triazole ring, str), 1468(s) (aliphatic $-\mathrm{C}-$ $\mathrm{CH}_{2}$-, bend), 1385(s) (aliphatic $-\mathrm{C}-\mathrm{CH}_{3}$, str), 1248(s) (Ar $-\mathrm{O}-\mathrm{CH}_{2}-$, str), 1197(s) (ester -C-O-, str), 1095(m) (-O- $\mathrm{CH}_{2}-$, bend), 842(s) (Ar para disubstitution, bend).

\section{${ }^{1} \mathbf{H}-\mathrm{NMR}\left[\mathrm{CDCl}_{3}\right] \mathbf{\delta p p m}$}

7.66-7.68(d,2H,ArH in biphenyl ring), 7.32-7.41(m,8H,ArH in benzene and biphenyl), 7.037.09(d,6H,ArH in benzene and biphenyl), 4.02-4.07(q,4H,-O- $\left.\mathbf{C H}_{2}-\right), 2.49,2.64\left(2 \mathrm{~s}, 6 \mathrm{H},-\mathbf{C H}_{3}\right.$ attached to $\mathrm{C}=\mathrm{N}-$ in [1,2,3]-triazole ring), $1.81-2.01\left(\mathrm{~m}, 4 \mathrm{H},-\mathrm{O}-\mathrm{CH}_{2}-\mathrm{CH}_{2}-\right), 1.50-1.51\left(\mathrm{~m}, 4 \mathrm{H},-\mathrm{CH}_{2}-\mathrm{CH}_{2}-\mathrm{CH}_{2}-\mathrm{CH}_{3}\right)$, $1.37-1.39\left(\mathrm{~m}, 8 \mathrm{H},-\mathrm{CH}_{2}-\mathrm{CH}_{2}-\mathrm{CH}_{3}\right), 0.88-0.94\left(\mathrm{t}, 6 \mathrm{H},-\mathrm{CH}_{2}-\mathrm{CH}_{2}-\mathrm{CH}_{3}\right)$.

The scheme of the synthesis is given below:

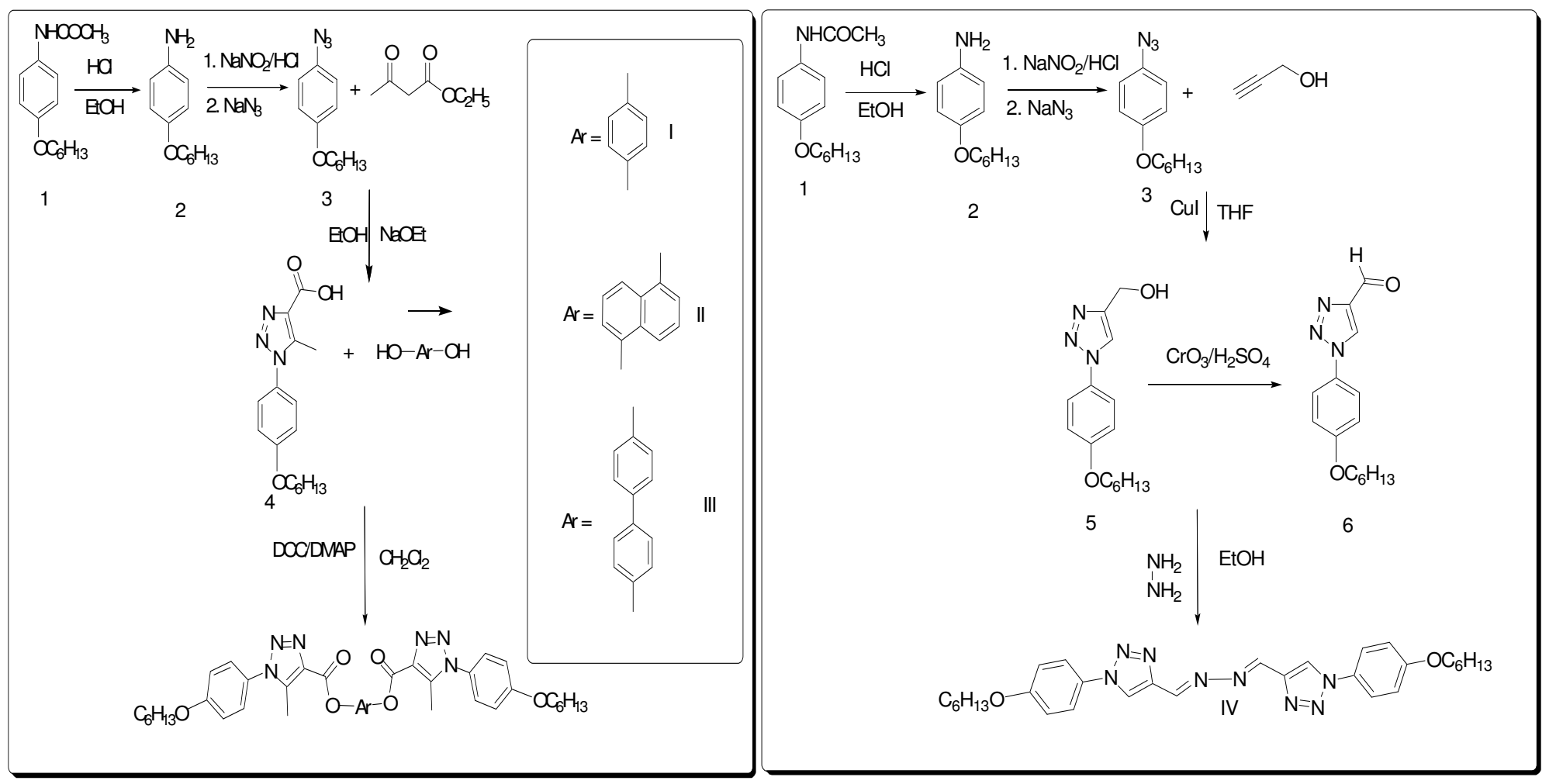

Scheme-1: Synthesis of compounds I, II, III and IV

\section{${ }^{13} \mathrm{C}-\mathrm{NMR}\left[500 \mathrm{MHz}, \mathrm{CDCl}_{3}\right] \delta \mathrm{ppm}$}

163.04(carbonyl carbon in ester), 160.45, 160.37(aromatic -C-O-), 160.19(-C-O- in biphenyl ring), 153.96, 149.88(quaternary carbon in benzyl ring), 140.26, 138.40(aromatic -C-N-), 137.73, 135.75, 128.25, 128.18(=C- in biphenyl ring), 127.87, 122.15(=C- in triazole ring), 126.75, 126.60, 115.34, 115.20(aromatic $=\mathbf{C H}-), 68.54,68.49\left(-\mathrm{O}-\mathrm{CH}_{2}-\right), 31.54,31.15\left(-\mathrm{O}-\mathrm{CH}_{2}-\mathrm{CH}_{2}-\right), 29.69,29.08\left(-\mathbf{C H}_{2}-\mathrm{CH}_{2}-\right.$ $\left.\mathrm{CH}_{2}-\mathrm{CH}_{3}\right), \quad 25.54, \quad 25.43\left(-\mathrm{CH}_{2}-\mathrm{CH}_{2}-\mathrm{CH}_{3}\right), \quad 22.59\left(-\mathrm{CH}_{2}-\mathrm{CH}_{3}\right), \quad 14.02\left(-\mathrm{CH}_{2}-\mathrm{CH}_{3}\right), \quad 10.06,9.95\left(-\mathrm{CH}_{3}\right.$ attached to triazole ring).

\section{RESULTS AND DISCUSSION}

The scheme of the preparation of 1,4-disubstituted triazole derivatives containing ester and Schiff's base as the center core are shown in scheme 1. 4-hydroxyl acetamido phenol is alkylated using hexyl bromide to get 4-hexyloxy acetamido phenol (1). The structure of (1) is confirmed with the peaks by FTIR:1240(s)(Ar $-\mathrm{O}-\mathrm{CH}_{2}-$, str), 1029(m) $\left(-\mathrm{O}-\mathrm{CH}_{2}-\right.$, bend), 830(s) (Ar para disubstitution, bend), by 
${ }^{1} \mathrm{HNMR} \delta(\mathrm{ppm}): 3.92-3.95\left(\mathrm{t}, 2 \mathrm{H},-\mathrm{O}-\mathrm{CH}_{2}-\right)$ and ${ }^{13} \mathrm{CNMR} \delta(\mathrm{ppm})$ : 68.31(-C-O-). This is further reduced by acid and alcohol to acquire the amine compound (2). Amine (2) is then diazotized by $\mathrm{NaNO}_{2}$ and the resultant product on treatment with sodium azide in acidic condition gives the 4-azido hexyloxy benzene (3). The structure of (3) is confirmed by a peak at $2111 \mathrm{~cm}^{-1}$ for $\mathrm{N}_{3}$ in FTIR spectra ${ }^{31}$. Refluxing of azide (3) with ethyl acetoacetate in sodium ethoxide yields 5-methyl-1-[4-hexyloxy phenyl]-1H-[1,2,3]triazole-4-carboxylic acid (4). The structure of (4) is confirmed by FTIR with the broad peak at $3448 \mathrm{~cm}^{-1}$ for $-\mathrm{OH}$ group, $1692 \mathrm{~cm}^{-1}$ for $-\mathrm{C}=\mathrm{O}$ group and $1515 \mathrm{~cm}^{-1}$ for $-\mathrm{N}=\mathrm{N}-$ in triazole ring ${ }^{32}$. In ${ }^{13} \mathrm{CNMR}$, the chemical shift at 135.76 and $127.79 \mathrm{ppm}$ confirms the presence of two $=\mathrm{C}$ - in triazole ring. The peaks at 7.35-7.38(d,2H,ArH), 7.05-7.08(d,2H,ArH) and 2.60(s,3H,- $\mathrm{CH}_{3}$ attached to $-\mathrm{CH}$ in [1,2,3]-triazole ring) is an supplementary evidence for the assigned structure ${ }^{32}$. The reaction of [1,2,3]-triazole acid derivative (4) with related diols (biphenyl diol, quinol and 1,5-naphthalene diol) is performed by means of dicyclohexylcarbodiimide in dichloromethane using dimethylamino pyridine as a catalyst. The final products I, II and III are recrystallised from ethanol. FTIR spectra show the disappearance of characteristic peaks of acid, diol and appearance of peaks at 1739, 1175 for (I), 1739, 1166 for (II) and 1744, $1197 \mathrm{~cm}^{-1}$ (III) in FTIR for $-\mathrm{C}=\mathrm{O}$ and $-\mathrm{C}-\mathrm{O}$ - respectively in ester group confirms the assigned structure. Further, in ${ }^{13} \mathrm{CNMR}$, the peaks at $160.45,160.46$ and $163.04 \mathrm{ppm}$ show a strong evidence for quaternary carbon in carbonyl group of ester in I, II and III.

The desired 1,4-disubstituted-[1,2,3]-triazole carbinol (5) is obtained by the 1,3-dipolar cycloaddition reaction of propargyl alcohol with o-hexyloxy phenyl azide (3). This sort of "click chemistry" reaction involving 1,3-dipolar cycloaddition of organic azides to triple bonds affords a 1/1 mixture of the 1,4- and 1,5-regioisomers ${ }^{11}$. The 1,4-regioisomer is selectively improved by using the $\mathrm{Cu}(\mathrm{I})$ or $\mathrm{Cu}(\mathrm{II})$ salts as a catalyst along with sodium ascorbate and water ${ }^{14,25,26}$. The best result in achieving 1,4-regioisomer (5) is accomplished by using a modified system with catalytic amount of copper(I) iodide and triethylamine(TEA). Based on the catalytic mechanism proposed by Sharpless et al. ${ }^{22}$, a concerted [2+3] cycloaddition through a stepwise process is achieved as an outcome of the terminal triple bond polarization by copper(I). Using this method, the regioisomer 1,4-disubstituted triazole carbinol (5) is synthesized and characterised by FTIR: 3221(s) (alcohol $-\mathrm{OH}, \mathrm{str}), 1050(\mathrm{~b})\left(1^{\circ}\right.$ alcohol), 3078(m) (Ar $\mathrm{C}-\mathrm{H}-$, str), $1517(\mathrm{~s})\left(-\mathrm{N}=\mathrm{N}-\right.$ in triazole ring, str), by ${ }^{1} \mathrm{HNMR} \delta(\mathrm{ppm}): 8.46(\mathrm{~s}, 1 \mathrm{H},-\mathrm{CH}$ of triazole ring), $4.88\left(\mathrm{~s}, 2 \mathrm{H},-\mathrm{CH}_{2}-\mathrm{OH}\right), 3.98-4.01\left(\mathrm{t}, 2 \mathrm{H}-\mathrm{O}-\mathrm{CH}_{2}-\right), 2.99\left(\mathrm{~s}, 1 \mathrm{H},-\mathrm{CH}_{2}-\mathrm{OH}\right)$. The crude product (5) obtained is converted into aldehyde (6) as per the reported procedure ${ }^{29,30}$ using Jones reagent and silica gel. The aldehyde (6) is confirmed by FTIR with a peak at $1690 \mathrm{~cm}^{-1}$ for carbonyl carbon in aldehyde, by ${ }^{1} \mathrm{HNMR}$ at $10.22 \mathrm{ppm}(\mathrm{s}, 1 \mathrm{H},-\mathrm{CHO})$, by ${ }^{13} \mathrm{CNMR}$ with a quaternary peak at $185.12 \mathrm{ppm}$ for carbonyl group in aldehyde of (6).

The aldehydic compound (6) on condensing with corresponding amino moieties (hydrazine hydrate) performed in p-toluene sulphonic acid as catalyst and ethanol as medium results in Schiff base (IV). The formation of an azomethine band causes a shift from 185.12 in (6) to $120.33,118.36 \mathrm{ppm}$ respectively for two such $-\mathrm{N}=\mathrm{CH}$ in IV resembles that of earlier works ${ }^{33}$. In ${ }^{1} \mathrm{HNMR}$ the shift of peak from $10.22 \mathrm{ppm}(\mathrm{s}, 1 \mathrm{H},-\mathrm{CHO})$ in (6) to $8.85 \mathrm{ppm}(\mathrm{s}, 2 \mathrm{H},-\mathrm{CH}=\mathrm{N}-)$ in IV confirms the proposed structure ${ }^{34}$. Moreover, the absence of a peak at 1690 (s) (aldehyde $-\mathrm{C}=\mathrm{O}$, str) and the presence of a peak at 1644(s) ($\mathrm{C}=\mathrm{N}-$, str) confirms the presence of $-\mathrm{N}=\mathrm{CH}$ - in Schiff's base ${ }^{31}$. An FTIR spectra for the compound IV is given in figure 1 and a sample ${ }^{1} \mathrm{HNMR}$ and ${ }^{13} \mathrm{CNMR}$ spectra of compound I are given in figure-2a and $2 b$.

The effects of varying the $\pi$ extended aromatic portion on the mesomorphic behavior is studied by synthesizing mesogens with different aromatic groups (Ar I, Ar II, Ar III) connected through an ester linkage to the $4^{\text {th }}$ position of the [1,2,3]-triazole ring. Their phase assignments, textures, and transition temperatures are studied by HOPM and DSC and their results are tabulated in Table-1. From the Table-1 it is clear that compound I having benzyl center unit presents short mesophase range $(\Delta T=77)$ than the compound III having biphenyl $(\Delta \mathrm{T}=92)$ in HOPM. Thus the stability of the mesophase increases with elongation of $\pi$ extended aromatic proton. This may be due to increase in length/breadth ratio and polarisabilty of the mesogenic unit. Representative polarising micrographs of the compound I, II, III and 
RASĀYAN J. Chem.

Vol. 10 | No. 1 |136-148 | January - March | 2017

IV are shown in fig.-4. The textures of the final compounds are identified and compared with previously observed mesophases.

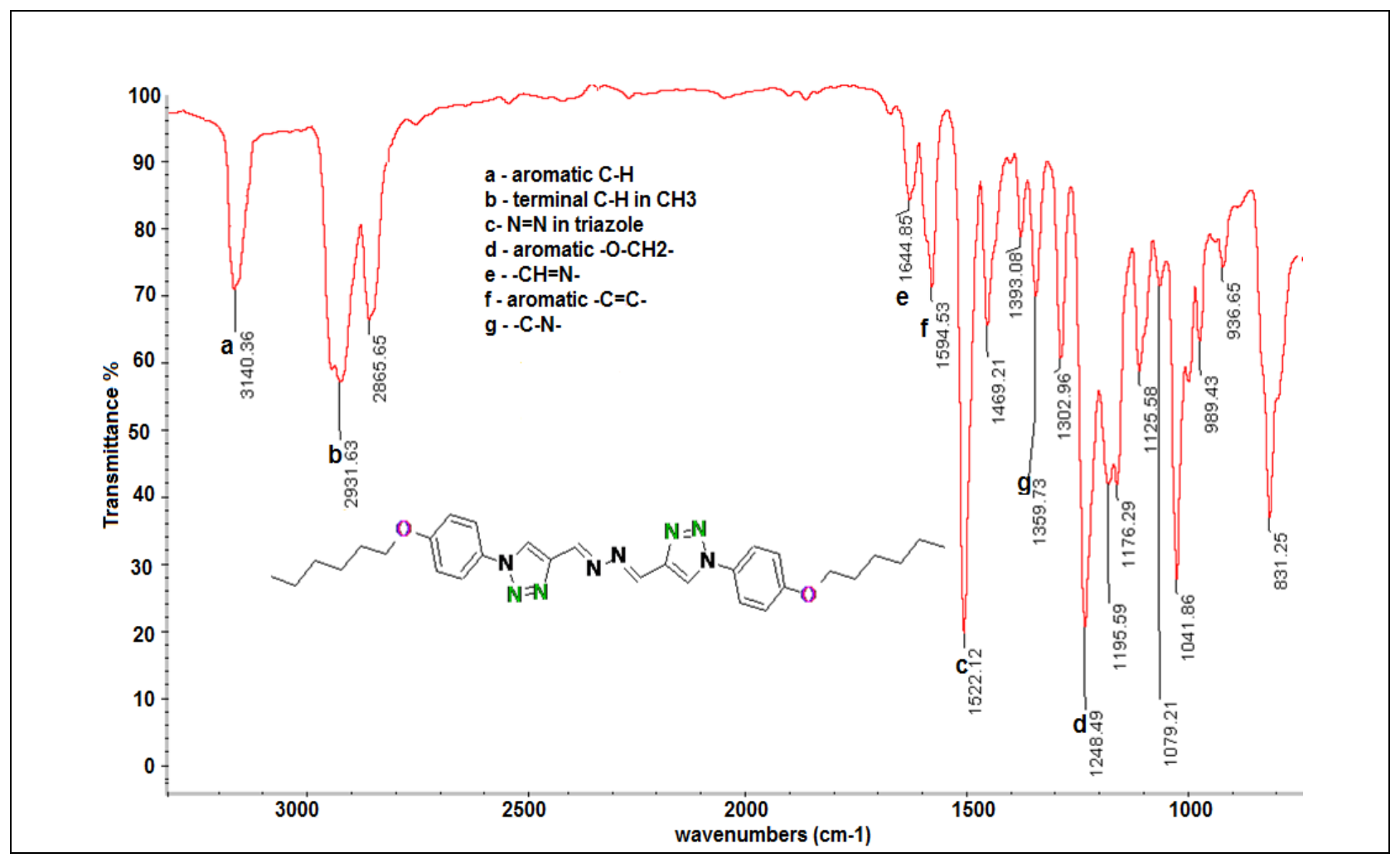

Fig.-1: FTIR spectra of compound IV

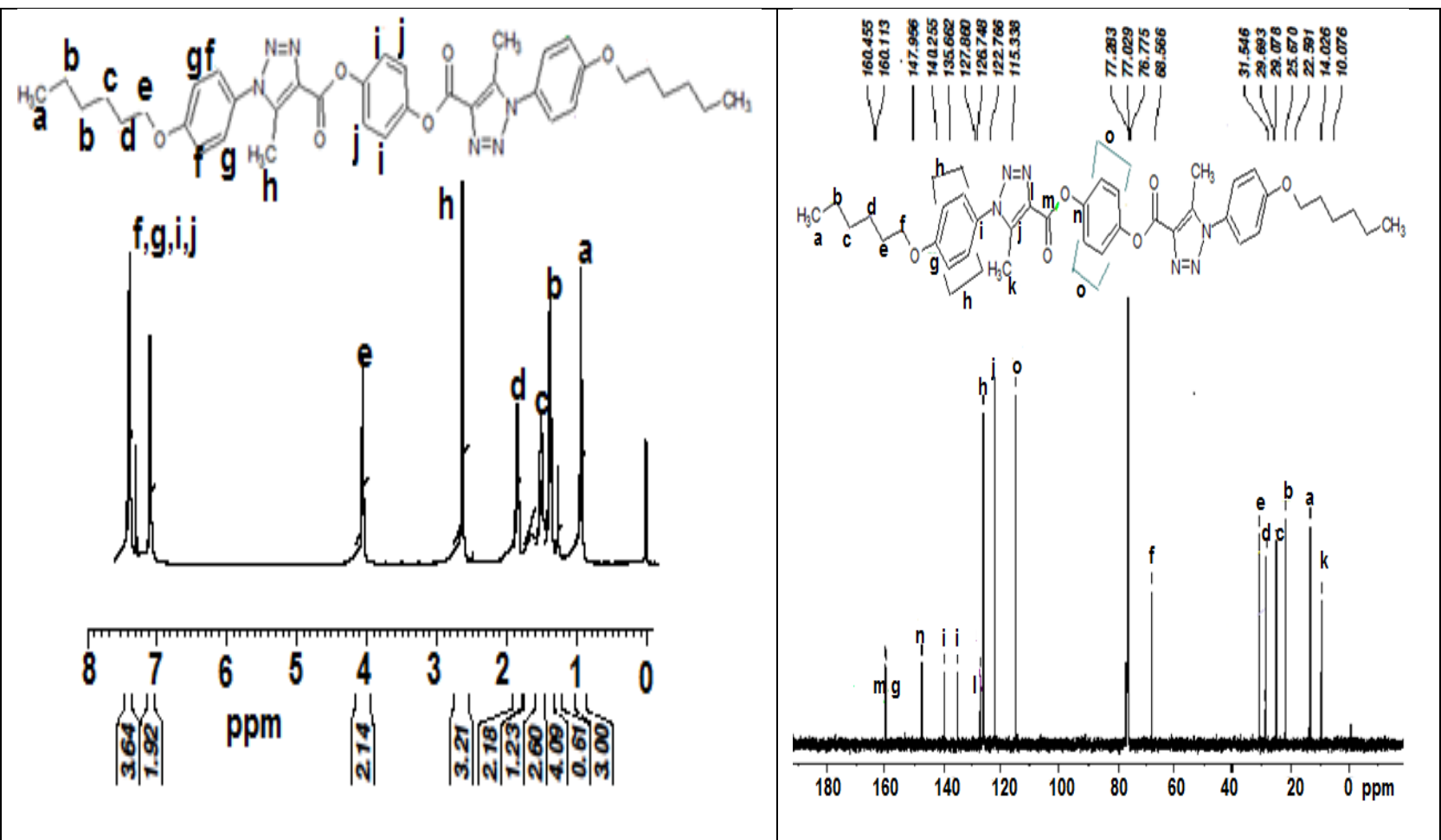

Fig.- 2a: ${ }^{1} \mathrm{HNMR}$ spectra of compound I

Fig.-2b: ${ }^{13} \mathrm{CNMR}$ of compound I 
RASĀYAN J. Chem.

Vol. 10 | No. 1 |136-148 | January - March | 2017

Table.-1: DSC and HOPM of target compounds I-IV

\begin{tabular}{c|c|c|c|c|c|c|c}
\hline Compound & \multirow{2}{*}{$\begin{array}{c}\text { Meso } \\
\text { phase }\end{array}$} & \multicolumn{2}{|c|}{ HOPM measurements in ${ }^{\circ} \mathrm{C}$} & \multicolumn{3}{c}{ DSC measurements in ${ }^{\circ} \mathrm{C}$} \\
\cline { 3 - 8 } & Cr-N & N-Iso & $\Delta \mathrm{T}$ & Cr-N & N-Iso & $\Delta \mathrm{T}$ \\
\hline I & Nematic & 158 & 235 & 77 & 156 & 231 & 73 \\
\hline II & Nematic & 180 & 260 & 80 & 178 & 257 & 79 \\
\hline III & Nematic & 178 & 270 & 92 & 175 & 268 & 93 \\
\hline IV & Nematic & 190 & 208 & 18 & 193 & 210 & 17 \\
\hline
\end{tabular}

The nematic phase for compounds I, II and III are confirmed by HOPM studies where a scheileren texture with dark points from which several black brushes originates (Fig.-4a, 4b, 4c) on heating. Furthermore, the thermal decomposition starts when they entry in 235,260 and $270^{\circ} \mathrm{C}$ during heating. Of these, the compound with biphenyl center core (III) has high melting point than compound with benzyl center unit (I) and naphthalene center unit (II). This may be due to the elongation of aromatic unit resulting in stability of mesophase.

The compound IV shows nematic phase with grainy texture (Fig - 4d) which may be due to increase in more and more segmental mobility of Schiff's base center core. The DSC of IV also confirms the same and on heating, the solid slowly transforms into mesophase at $193^{\circ} \mathrm{C}$ and on further heating, the thermal decomposition starts after $210^{\circ} \mathrm{C}$. Figure-3 exemplifies this profile.

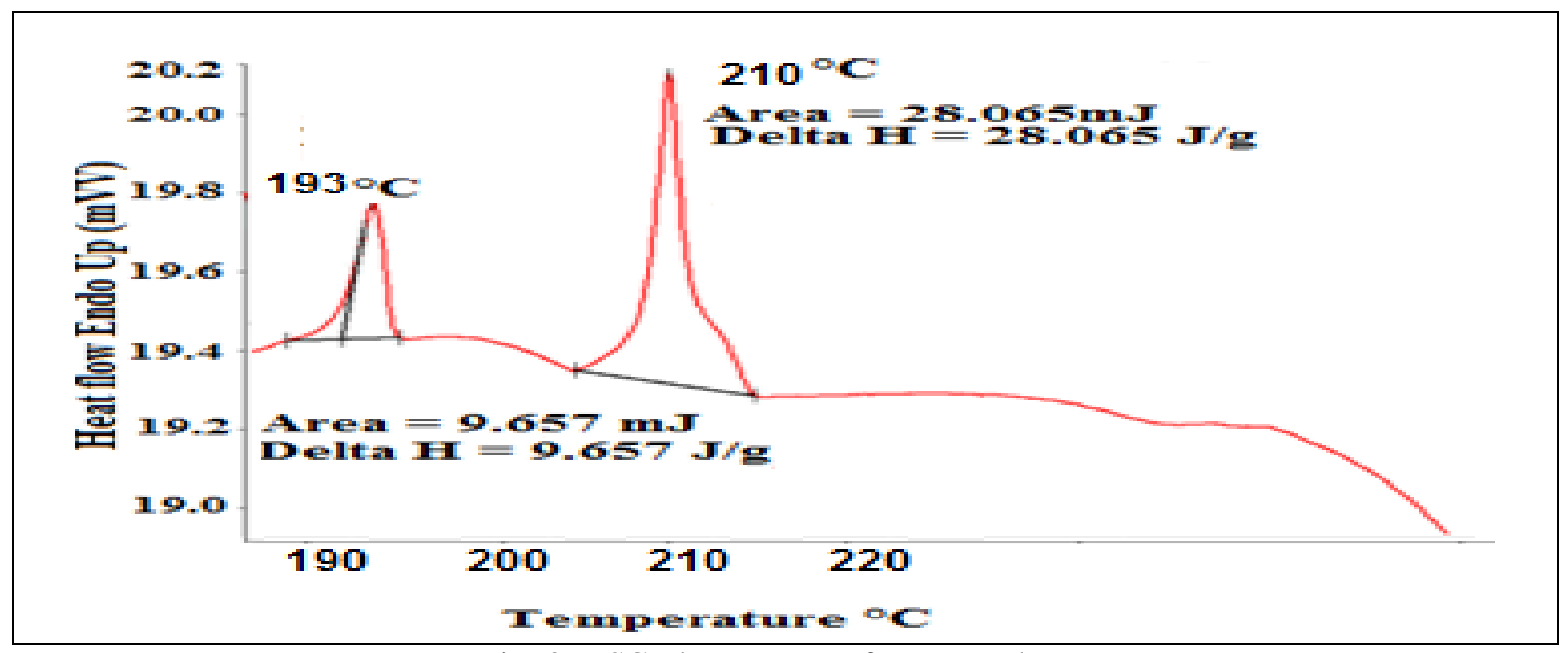

Fig.-3: DSC Thermogram of compound IV

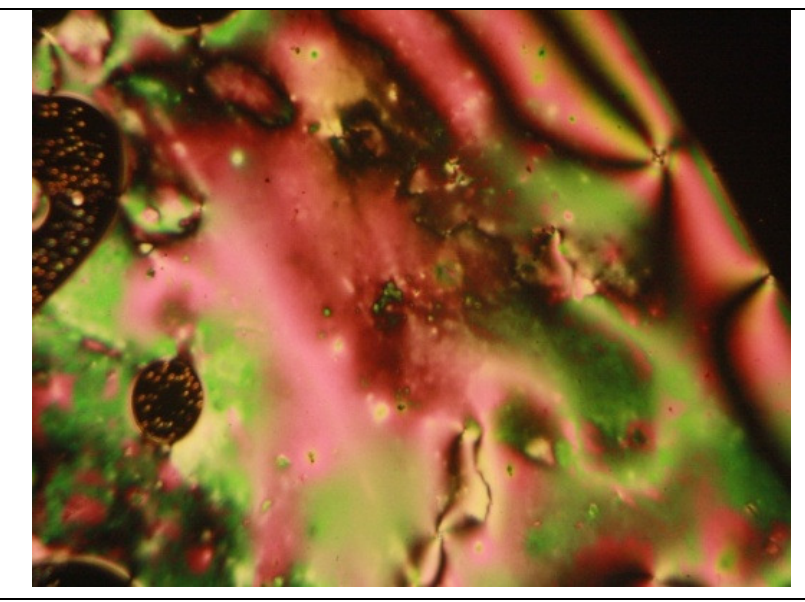

Fig.-4a: Nematic of compound I

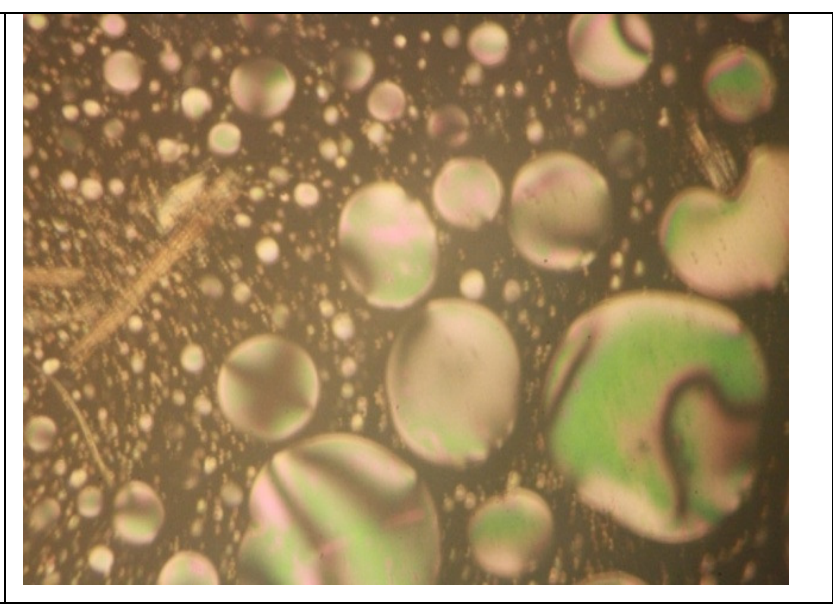

Fig.-4b: Nematic of compound II 
RASĀYAN $J$. Chem.

Vol. 10 | No. 1 |136-148 | January - March | 2017

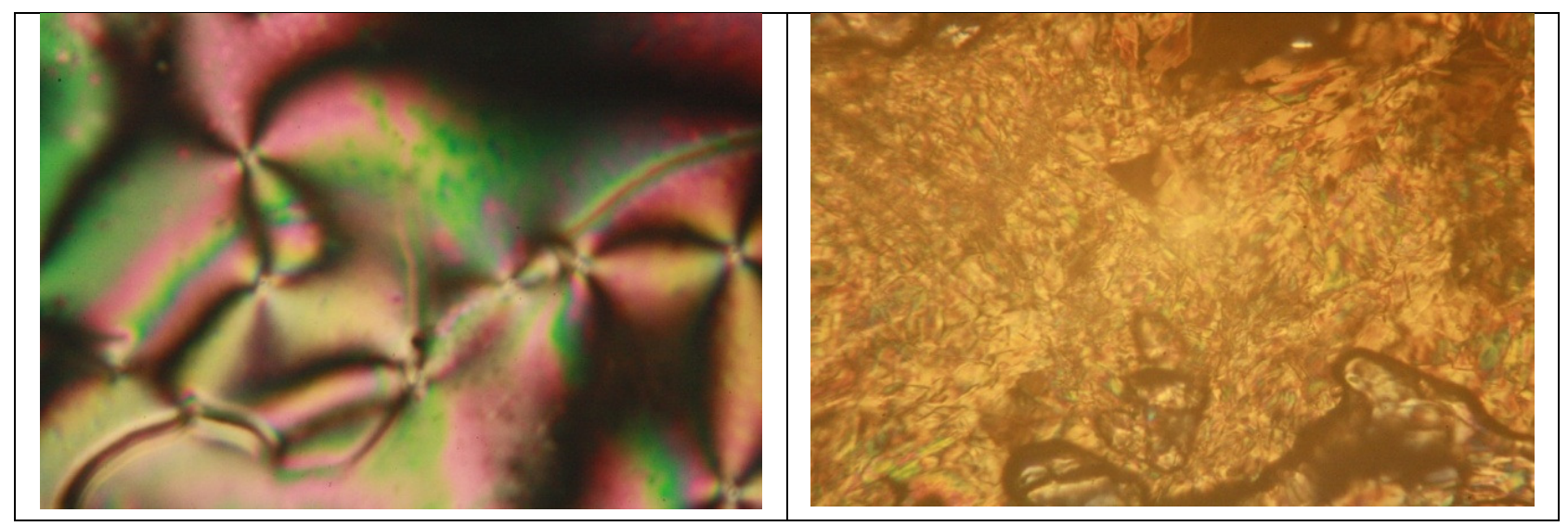

Fig.-4c: Nematic of compound III

Fig.-4d: Nematic of compound IV
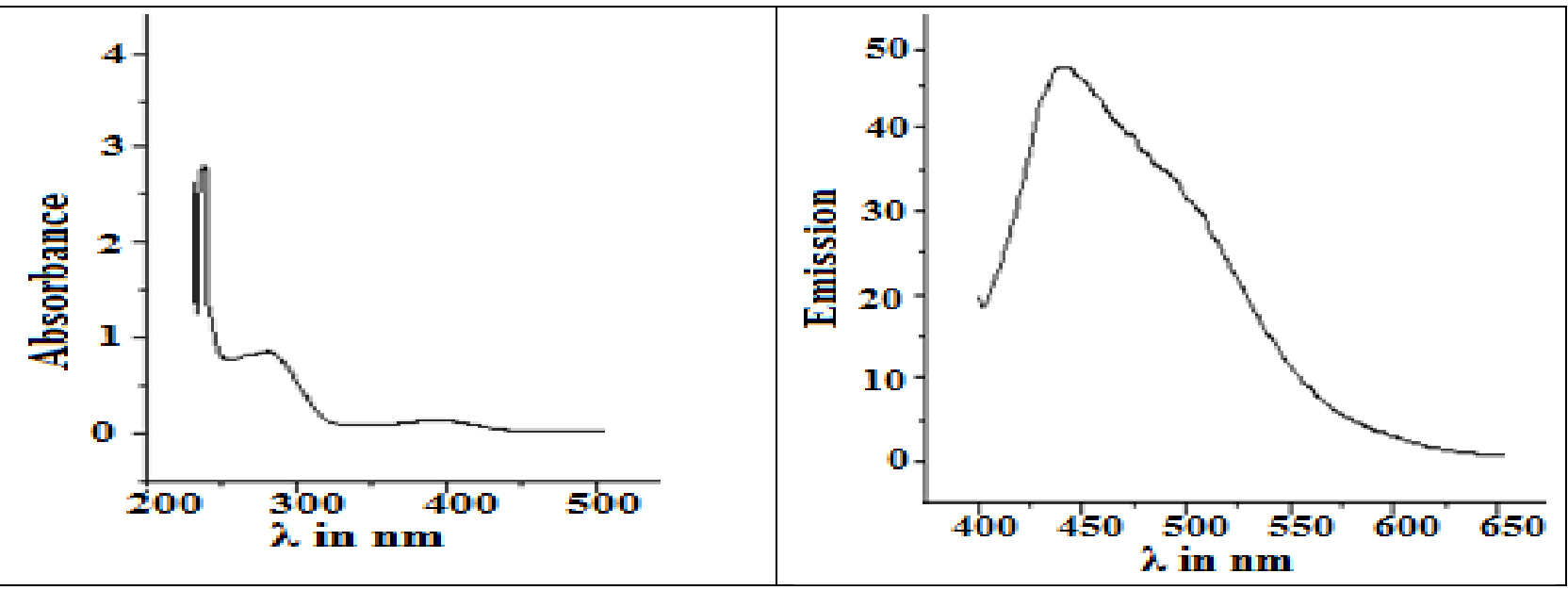

Fig. -5a: UV absorption spectra of compound I

Fig.-5b: UV emission spectra of compound I

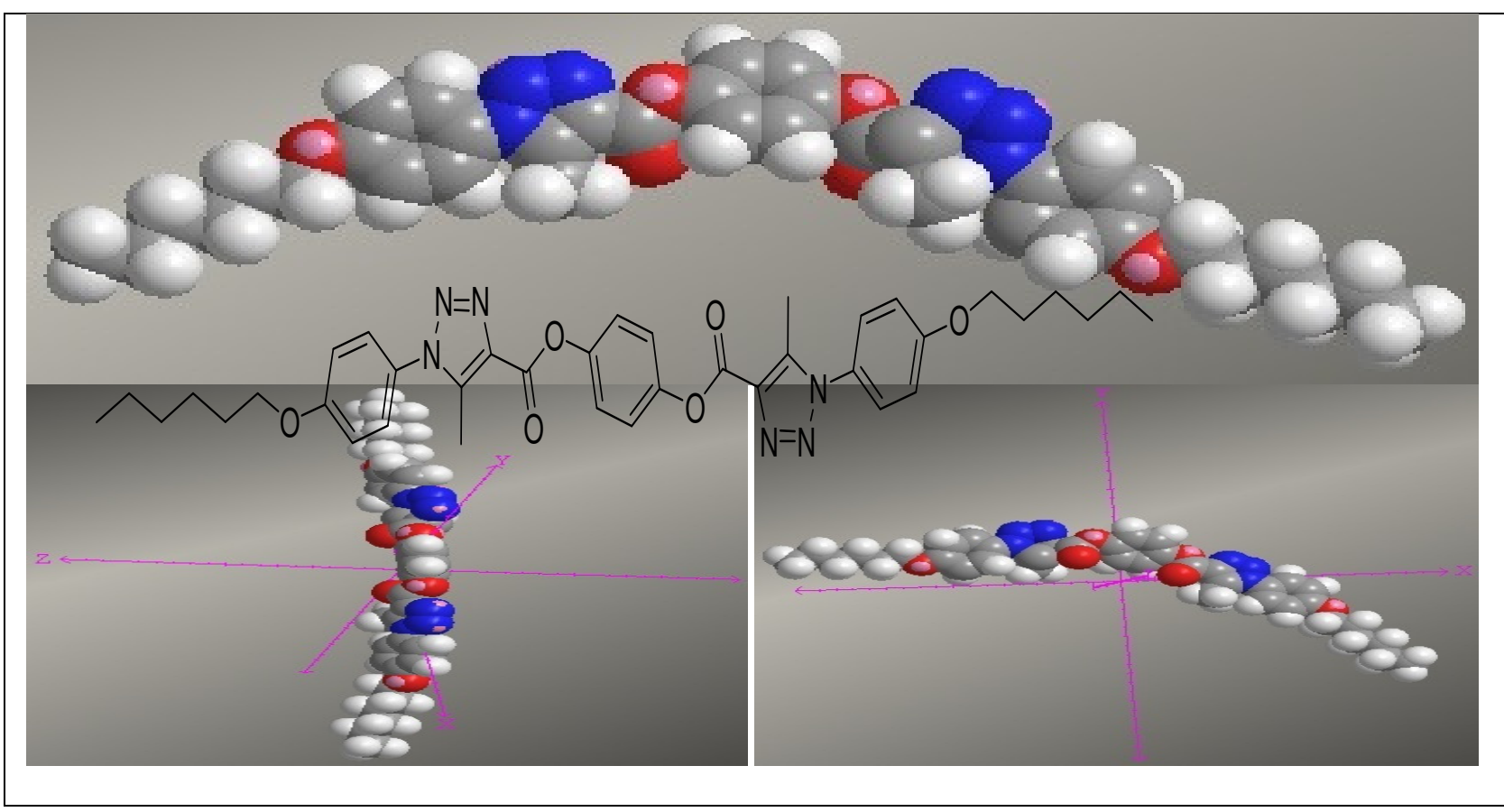

Fig.-6a: Energy minimized structure of compound I 


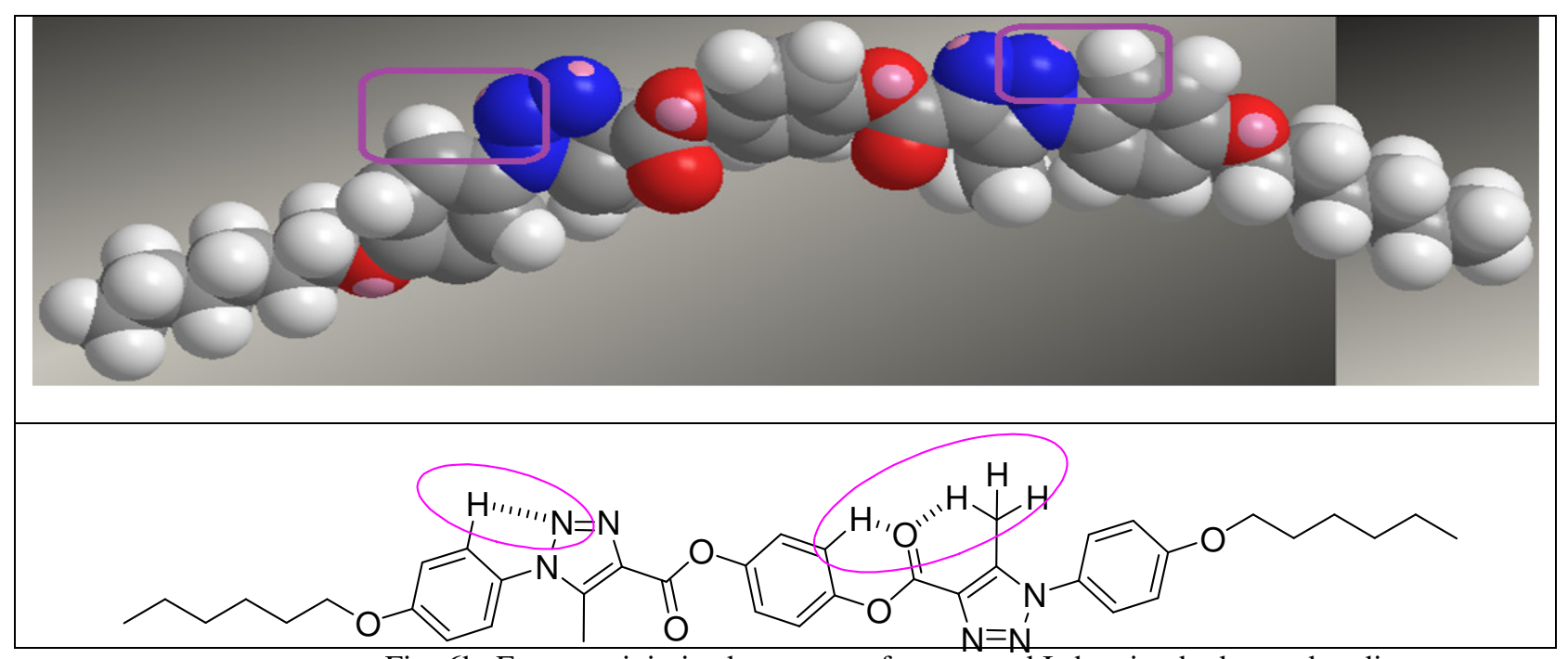

Fig.-6b: Energy minimized structure of compound I showing hydrogen bonding

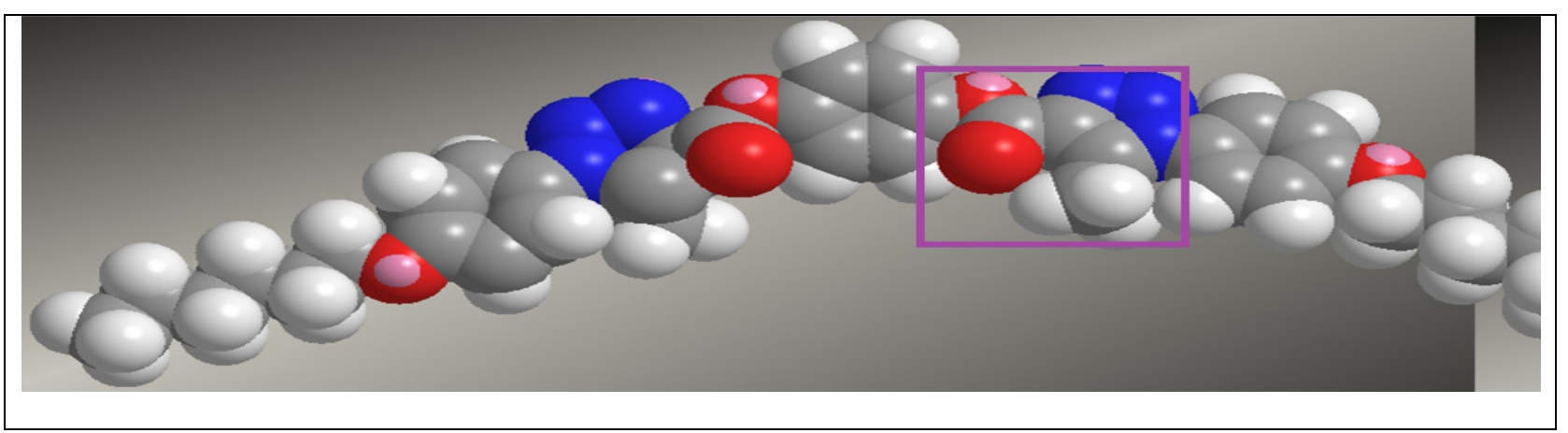

Fig.-6c: Energy minimized structure of compound I showing crowded kink effect

The target compounds reveal a weak fluorescence band near blue region with a maximum of $447 \mathrm{~nm}$ while the maximum of the absorption is close to 250nm (Fig 5a and Fig 5b). A small stokes shift of this weak fluorescence may be due to the two possible intramolecular hydrogen bonding. One is between the nitrogen of the heterocyclic ring and hydrogen of an aromatic ring attached to the first nitrogen of the $[1,2,3]$-triazole ring ${ }^{35}$. Moreover, another marked hydrogen bonding is between the oxygen of ester group, hydrogen of an aromatic ring attached to $1^{\text {st }}$ position of the [1,2,3]-triazole ring and methyl hydrogen attached to $4^{\text {th }}$ position of $[1,2,3]$-triazole. Fig. $-6 \mathrm{a}$ and $6 \mathrm{~b}$ clearly depicts the energy minimized structure and intramolecular hydrogen bonding of compound $\mathrm{I}$.

The compound that contains liquid crystallinity usually consists of comparatively rigid structures that confer high extension on the molecules. Polarisability of the molecules and their constituent groups rendering intramolecular attraction may contribute to the stability of the mesophase. Thus in the molecule, the additional motion is possible because of the parallel configuration of the angular bridging ester group. Moreover, the ester linkage gives a sideways displacement keeping the 1,4 axes of the center unit parallel and thus facilitating the kink effect ${ }^{36}$. The pictures of fig $6 \mathrm{c}$ clearly epitomize the kink effect.

\section{CONCLUSION}

The 1,4-disubstituted triazole derivatives detailed here represents novel liquid crystals containing ester and Schiff's base as linking units. FTIR, ${ }^{1} \mathrm{HNMR}$ and ${ }^{13} \mathrm{CNMR}$ confirm the proposed structure for intermediates and target compounds. Polarized microscopic investigation reveals that all the compounds show liquid crystalline properties with nematic texture. Further investigation with DSC measurements 
shows two endothermic peaks confirming the mesophase formation. The targeted and synthesized compounds structure are varied by adopting different linking moieties between two 1,4-disubstituted triazole derivatives taking into consideration that the appearance of mesomorphic behavior depends on length/diameter ratio of the molecules. The ester and imine linking units establish the increase of polarities, keeping the linear pattern, whereas the terminal chains balance rigidity with the flexibility of molecule. From the above details, the structure and mesomorphous properties of 1,4-disubstituted-[1,2,3]triazole derivatives are related and that these compounds have a nematic liquid crystalline phase. The weak fluorescence may be due to the hydrogen bond type of interaction between ortho hydrogen of the phenyl group and nitrogen of triazole ring.

\section{REFERENCES}

1. Franklin D. Saeva, Liquid Crystals: The Fourth State of Matter, Marcel Dekker, New York (1979).

2. R. Cristiano, D. M.Santos, G. Conte and H. Gallardo, Liq. Cryst, 33, 997 (2006).

3. D. Demus, J. W. Goodby, G. W. Gray, H. W. Spiess and V. Vill, Handbook of Liquid Crystals, Wiley-VCH, Weinheim (1998).

4. H. Gallardo and L. Maurmann, Mol. Cryst. Liq. Cryst, 378, 23 (2002).

5. H. Gallardo and F. C. Silva, J. Chem. Soc, Perkin Trans. 3, 319 (1987).

6. T. Kato, N. Mizoshita and K. Kishimoto, Angew. Chem. Int. Ed, 45, 38 (2006).

7. Valery P. Shibaev and Lui Lam, Liquid Crystalline and Mesomorphic Polymers, Springer-Verlag, New York, p.26 (1994).

8. H. Zaschke, Wiss. Z. Univ, 3, 35, (1980).

9. J. W. Brown and D. J. Byron, Mol. Cryst. Liq. Cryst, 173, 121 (1989).

10. R. Iglesias, J. L. Serrano and T. Sierra, Liq. Cryst, 321, 643 (1997).

11. H. Gallardo, A. J. Bortoluzzi and D. M. Santos, Liq. Cryst, 35, 719 (2008).

12. R. Ranjan and W. J. Brittain, Macromolecules, 40, 6217, (2007).

13. Peng wu and V. Fokin, Aldrichimica Acta, 7, 40 (2007).

14. P. Appukkuttan, W. Dehaen, V. V. Fokin and E. V. D. Eycken, Org. Lett, 6, 4223 (2004).

15. E. Uchida and N. Kawatsuki, Polymer, 47, 2322 (2006).

16. R. Manetsch, A. Krasinski, Z. Radic, J. Raushel, P. Taylor, K. B. Sharpless and H. C. Kolb, J. Am. Chem. Soc, 126, 12809 (2004).

17. H. C. Kolb and K. B. Sharpless, Drug Discovery Today, 8, 1128 (2003).

18. U. V. Vasconcelos, E. Dalmolin and A. A. Merlo, Org. Lett, 7, 1027 (2005).

19. R. Cristiano, F. Ely and H. Gallardo, Liq. Cryst, 32, 15 (2005).

20. A. J. Paraskos and T. M. Swager, Chem. Mater, 14, 4543 (2002).

21. B.Y. Zhang, Y. G. Jia, D. S. Yao and X. W. Dong, Liq. Cryst, 31, 339 (2004).

22. H. C. Kolb, M. G. Finn and K. B. Sharpless, Angew. Chem, 40, 2004 (2001).

23. P. J. Colling and M. Hird, Introduction to liquid crystals: chemistry and physics, Taylor \& Francis Ltd, Bristol, (1998).

24. R. Huisgen, Angew. Chem. Int. Ed, 2, 565 (1963).

25. V. V. Rostovtsev, L. G. Green, V. V. Fokin and K. B. Sharpless, Angew. Chem. Int. Ed, 41, 2596 (2002).

26. C. W. Tornoe, C. Christensen and M. Meldal, J.Org. Chem, 67, 3057 (2002).

27. H. Fahmi, T. Lovell, R. Hilgraf, V. V. Rostovtsev, L. Noodleman, K. B. Sharples and V. V. Fokin, J. Am. Chem. Soc, 127, 210 (2005).

28. D. Srividhya, S. Manjunathan, S. Nithyanandan, S. Balamurugan and S. Senthil, Chinese J. Polym. Sci, 27,761 (2009).

29. Ji-Dong lou, Yi-chun Ma and Chun-Ling Gao, Synthesis And Reactivity In Inorganic, MetalOrganic And Nano-Metal Chemistry, 36, 381 (2006).

30. G. Cainelli and G. Cardillo, Chromium Oxidations in Organic Chemistry, Springer - Verlag, Berlin, p.118 (1984).

31. Huda Ahmed Hassan, J. Al-Nahrain univ, 16, 53 (2013). 
RASĀYAN J. Chem.

Vol. 10 | No. 1 |136-148 | January - March | 2017

32. Sagar Narala, Venkateswararrao Jukanti and A.K.S.Bhujanga rao, Asian J. Pharm. Clin. Res, 5, 89 (2012).

33. I. Gurol and V. Ahsen, Mol. Cryst. Liq. Cryst, 442, 103 (2005).

34. B. T. Thaker, D. M. Patel and P. K. Tandel, Indian J. Chem, 46, 1020 (2007).

35. A. A. Vieira, R. Cristiano, A. J. Bortoluzzi and H. Gallardo, J. Mol. Struct, 875, 364 (2008).

36. Chung and Tai-shung, Thermotropic liquid crystal polymers: thin film polymerisation, characterisation blends and applications, Technomic Pub. Co., Lancaster, Pa., (2001).

[RJC-1576/2017] 